\title{
Cover page for the Special Issue on: Parametric CAD Modeling for Naval Architecture, Ocean \& Marine Engineering (NAOME)
}

\author{
GUEST EDITOR: P.D. Kaklis ${ }^{\mathrm{a}}$ \\ ${ }^{a}$ Department of Naval Architecture, Ocean and Marine Engineering \\ University of Strathclyde, Glasgow, UK
}

\section{Cover Page}

The motivating aim of the present Special Issue (SI) is to attract the interest of researchers who are active in the area of Parametric Modeling (PM) for Naval Architecture, Ocean \& Marine Engineering (NAOME) and project it onto the broader reader audience of Ocean Engineering, Elsevier, through a number of carefully reviewed papers. We strongly believe that, as the need of the NAOME industrial and academic communities for optimising complex shapes increases persistently, the availability of efficient and robust parametric modelers of the involved geometric environments will be an increasingly important factor for successful designs and constructions.

The favorable acceptance of the SI Call for Papers and the quality of the works composing the current issue are indicators that our initial objectives are more-or-less fulifilled. Furthermore, the versatility of the methods and the wide range of application areas dealt with should convince the OE readers that PM is a research area with strong potential for further development. This expansion can be even more boosted if the interested NAOME-research communities culitivate stronger links with:

- Emerging technologies in the areas of CAD representations,

- Novel computational methods that can be seamlessly integrated with the processing of the underlying geometries and

- Dimensionality reduction for fast exploration of design spaces.

The application areas covered in the present SI include:

- Unmanned surface vessels[1],

- SWATHs 2,

- Yacht hulls 3],

- Ro-Ro, 4], and Ro-PAX [5] ships,

- Marine propellers [6] and hydrofoils [7.

We also provide the reader with:

- A state-of-the-art paper [8] on simulation-driven design as encountered in the maritime industry.

\footnotetext{
${ }^{*}$ Corresponding author: panagiotis.kaklis@strath.ac.uk
} 
In addition, the paper addresses potential solutions for faster turn-around times via dimensionality reduction and exploitation of gradient information as derived from adjoint simulations. In a relevant context, the methodology developed in $[9]$ is capable to:

- Assess different design spaces and shape parameterization methods before optimization is performed and without the need of running simulations for the performance prediction, and

- Reduce the dimensionality of the design space by developing an appropriate shape reparameterization.

Finally, two papers are focusing on the geometrical aspects of PM for ship design:

- In 10 the authors exploit a dimensionality-reduction approach, referred to as Reduced Order Models (ROMs), to create a parametric model for both global and local hull shape variations. For this purpose, a geometric transformation relying on the so-called Proper Orthogonal Decomposition (POD) method is applied on top of a combined subdivision - FFD (Free Form Deformation) technique conceived for modeling and variation of hull shapes.

- In 11] a novel CAD representation is employed, apparently for the first time, for developing a T-splines-based parametric modeller, TshipPM(c), capable to provide smooth geometries for complex ship forms at a low cost in comparison with parametric modellers employing the standard NURBS representation.

\section{References}

[1] "Automatic Optimal Design of Self-righting Deck of USV based on Combined Optimization Strategy" by Guan Guan, Lei Wang, Jiahong Geng and Qu Yang; School of Naval Architecture, Dalian University of Technology, Dalian, China; https://doi.org/10.1016/j.oceaneng. 2020.107824

[2] "A Parametric Methodology for the Preliminary Design of SWATH hulls" by F. Pérez-Arribas and J. Calderon-Sanchez; Universidad Politécnica de Madrid (UPM), Madrid, Spain; https: //doi.org/10.1016/j.oceaneng.2019.106823.

[3] "GenYacht: An Interactive Generative Design System for Computer-Aided Yacht Hull Design" by Shahroz Khan [1], Erkan Gunpinar [2] and Bekir Sener [3]; [1] University of Strathclyde, Glasgow, UK, [2] Istanbul Technical University, Istanbul, Turkey, [3] Yildiz Technical University, Istanbul, Turkey; https://doi.org/10.1016/j.oceaneng.2019.106462.

[4] "Parametric Design and Optimisation of High-Speed Ro-Ro Passenger Ships" by Sotiris Skoupas [1], George Zaraphonitis [2] and and Apostolos Papanikolaou [3]; [1] Lloyd's Registe, Piraeus, Greece, [2] National Technical University of Athens, Athens, Greece, [3] Hamburger Schiffbau-Versuchsanstalt, Hamburg, Germany; https://doi.org/10.1016/j.oceaneng. 2019.106346

[5] "Multi-objective robust early stage ship design optimisation under uncertainty utilising surrogate models", Alexandros Priftis [1], Evangelos Boulougouris [1], Osman Turan [2], Georgios Atzampos [1]; [1] Maritime Safety Research Centre, University of Strathclyde, [2] University of Strathclyde, Glasgow, UK; https://doi.org/10.1016/j.oceaneng.2019.106850.

[6] "Parametric models for marine propellers" by A. Arapakopoulos [1], R. Polichshuk [2], Z. Segizbayev [2], S. Ospanov [2], A.I. Ginnis [1], and K.V. Kostas [2]; [1] School of Naval Architecture \& Marine Engineering, National Technical University of Athens, Greece, [2] Department of Mechanical \& Aerospace Engineering, Nazarbayev University, Kazakhstan; https://doi.org/10.1016/j.oceaneng.2019.106595. 
[7] "Parametric model for the reconstruction and representation of hydrofoils and airfoils", K.V. Kostas [1], A. Amiralin [1], S. Sagimbayev [1], T. Massalov [1], Y. Kalel [1] and C.G. Politis [2]; [1] Department of Mechanical \& Aerospace Engineering, Nazarbayev University, Kazakhstan, [2] Department of Naval Architecture, University of West Attica, Greece; https://doi.org/ $10.1016 /$ j. oceaneng. 2020.107020

[8] "Faster turn-around times for the design and optimization of functional surfaces", S. Harries and C. Abt, FRIENDSHIP SYSTEMS AG, Germany; https://doi.org/10.1016/j. oceaneng.2019.106470

[9] "Design-space Assessment and Dimensionality Reduction: An Off-line Method for Shape Reparameterization in Simulation-based Optimization", Danny D'Agostinoa [1],[2], Andrea Serani [1] and Matteo Diez [1]; CNR-INM, National Research Council-Institute of Marine Engineering, Rome, Italy, [2] Dept. of Computer, Control, and Management Engineering "A. Ruberti", Sapienza University of Rome, Italy; https://doi.org/10.1016/j .oceaneng. 2019.106852

[10] "Parametric hull shape variations by Reduced Order Model based geometric transformation", Antonio Coppede, Stefano Gaggero, Giuliano Vernengo1 and Diego Villa, Dept. of Electric, Electronic and Telecommunication Engineering, and Naval Architecture (DITEN), University of Genoa, Italy; https://doi.org/10.1016/j.oceaneng.2020.107826

[11] “A T-splines-based Parametric Modeller for Computer-Aided Ship Design", T. Katsoulis, X. Wang, P.D. Kaklis, University of Strathclyde Department of Naval Architecture, Ocean and Marine Engineering (NAOME), Glasgow, UK; https://doi.org/10.1016/j . oceaneng. 2019.106433 